\title{
Reduced capacity of alternative $\sigma s$ to melt promoters ensures stringent promoter recognition
}

\author{
Byoung-Mo Koo, ${ }^{1}$ Virgil A. Rhodius, ${ }^{1}$ Gen Nonaka, ${ }^{1,5}$ Pieter L. deHaseth, ${ }^{2,3}$ and Carol A. Gross ${ }^{1,4,6}$ \\ ${ }^{1}$ Department of Microbiology and Immunology, University of California at San Francisco, San Francisco, California 94158, USA; \\ ${ }^{2}$ Center for RNA Molecular Biology, Case Western Reserve University, Cleveland, Ohio 44106, USA; ${ }^{3}$ Department of \\ Biochemistry, Case Western Reserve University, Cleveland, Ohio 44106, USA; ${ }^{4}$ Department of Cell and Tissue Biology, \\ University of California at San Francisco, San Francisco, California 94158, USA
}

\begin{abstract}
In bacteria, multiple $\sigma s$ direct RNA polymerase to distinct sets of promoters. Housekeeping $\sigma s$ direct transcription from thousands of promoters, whereas most alternative $\sigma$ s are more selective, recognizing more highly conserved promoter motifs. For $\sigma^{32}$ and $\sigma^{28}$, two Escherichia coli Group $3 \sigma \mathrm{s}$, altering a few residues in Region 2.3, the portion of $\sigma$ implicated in promoter melting, to those universally conserved in housekeeping $\sigma s$ relaxed their stringent promoter requirements and significantly enhanced melting of suboptimal promoters. All Group 3 os and the more divergent Group 4 os have nonconserved amino acids at these positions and rarely transcribe $>100$ promoters. We suggest that the balance of "melting" and "recognition" functions of $\sigma \mathrm{s}$ is critical to setting the stringency of promoter recognition. Divergent $\sigma s$ may generally use a nonoptimal Region 2.3 to increase promoter stringency, enabling them to mount a focused response to altered conditions.
\end{abstract}

[Keywords: $\sigma$ factor; Region 2.3; melting proficiency; promoter stringency]

Supplemental material is available at http://www.genesdev.org.

Received July 14, 2009; revised version accepted August 31, 2009.

In bacteria, promoter recognition is accomplished primarily by the $\sigma$ subunit of RNA polymerase (RNAP). A single housekeeping $\sigma$ directs RNAP to thousands of promoters, whereas alternative $\sigma$ s generally orchestrate transcription from substantially fewer promoters, allowing focused responses to cellular, environmental, and developmental signals (Gruber and Gross 2003; Paget and Helmann 2003; Gama-Castro et al. 2008). This unequal division of labor requires a housekeeping $\sigma$ with broad, flexible promoter recognition (Hook-Barnard and Hinton 2007), and alternative $\sigma$ s with more restricted recognition (Amaya et al. 2001; Britton et al. 2002; Eichenberger et al. 2003; Nonaka et al. 2006; van Schaik et al. 2007; Zhao et al. 2007; Asayama and Imamura 2008; Koo et al. 2009a,b). More extensive use of activators and repressors by the housekeeping $\sigma$ than by alternative $\sigma \mathrm{s}$ partially explains this distinction (Browning and Busby 2004). Here, we provide evidence for an additional strategy intrinsic to the $\sigma \mathrm{s}$ themselves, demonstrating that some alternative $\sigma$ s are specifically constructed to limit their ability to transcribe a wide range of promoters.

\footnotetext{
${ }^{5}$ Present address: Ajinomoto Co., Inc., 15-1, Kyobashi 1-chome, Chuoku, Tokyo 104-8315, Japan

${ }^{6}$ Corresponding author.

E-MAIL cgross@cgl.ucsf.edu; FAX (415) 514-4080.

Article is online at http://www.genesdev.org/cgi/doi/10.1101/gad.1843709.
}

$\sigma s$ are sequence-specific DNA-binding proteins with a modular architecture, consisting of globular domains (Murakami and Darst 2003) subdivided into conserved regions (Fig. 1A). In free $\sigma$, DNA-binding determinants are masked by domain interactions (Dombroski et al. 1993; Sorenson and Darst 2006). However, strong interactions between RNAP and domains 2-4 $\left(\sigma_{2-4}\right)$ expose DNAbinding determinants and position domains for interaction with promoter motifs (Fig. 1A; Kuznedelov et al. 2002; Murakami et al. 2002). $\sigma_{4}$ recognizes the -35 motif (Gardella et al. 1989; Siegele et al. 1989), $\sigma_{3}$ recognizes the extended -10 (E-10) motif (Barne et al. 1997; Koo et al. $2009 \mathrm{a}, \mathrm{b})$, and $\sigma_{2}$ facilitates strand opening by three sequential activities: (1) recognition of the -10 and discriminator regions (Siegele et al. 1989; Daniels et al. 1990; Waldburger et al. 1990; Tatti et al. 1991; Feklistov et al. 2006; Haugen et al. 2006; Koo et al. 2009a,b), (2) participation in melting (Juang and Helmann 1994; Fenton et al. 2000; Tomsic et al. 2001; Lee and Gralla 2003), and (3) interaction with the -10 region nontemplate strand DNA to stabilize the melted state (Helmann and deHaseth 1999; Schroeder et al. 2009). $\sigma_{1}$ (Region 1.1) is unique to housekeeping $\sigma \mathrm{s}$ and has regulatory roles (Dombroski et al. 1992, 1993; Hook-Barnard and Hinton 2009).

The $\sigma^{70}$ superfamily includes three subfamilies, divided according to phylogenetic relatedness to the essential 
A

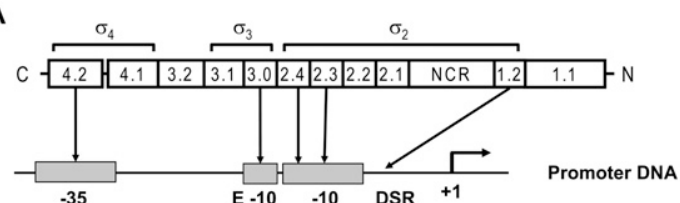

B

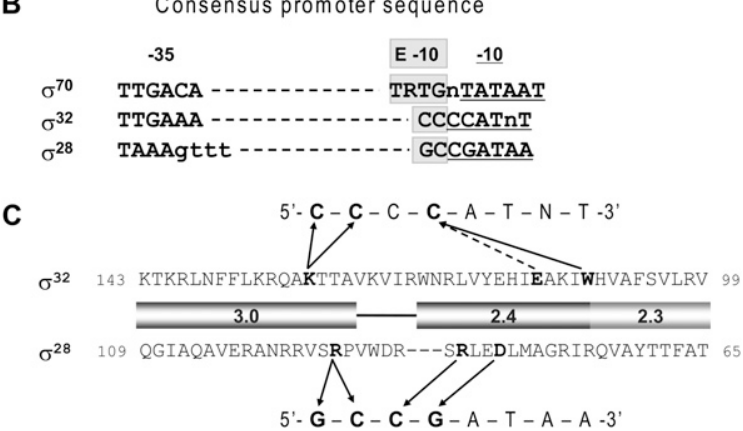

Figure 1. Promoter utilization by $\sigma .(A)$ Domain organization and conserved regions of $\sigma$ s and their functional assignments based on those of $\sigma^{70}$. A schematic of a typical promoter with conserved DNA regions $-35,-10$, and E-10, and discriminator (DSR) region from the transcription start site is shown below the linear representation of $\sigma$. Interactions between conserved region of $\sigma$ and promoter regions are indicated by arrows. In $\sigma_{2}$, Region 2.4 is implicated in both duplex recognition and interaction with nontemplate strand, Region 2.3 is implicated in duplex recognition and melting, and Region 1.2 recognizes the discriminator. The crystallized domains of $\sigma$ are indicated above the linear representation of $\sigma .(B)$ Consensus promoter sequences of $\sigma^{70}, \sigma^{32}$, and $\sigma^{28}$. E-10 motifs of each promoter are boxed in gray, and the -10 motifs of each promoter are underlined. $(C)$ Recognition of the -10 region of promoters by $\sigma^{32}$ and $\sigma^{28}$. Amino acid sequences of Regions 2.3, 2.4, and 3.0 of $\sigma^{32}$ and $\sigma^{28}$ are shown. The -10 consensus promoter sequences for each $\sigma$ are indicated above or below the amino acid sequences. Contacts between $\sigma$ and promoter are indicated by arrows. Solid arrows indicate direct contacts, and the arrow with the dotted line indicates contact to increase selectivity (Koo et al. 2009a,b).

housekeeping (Group 1) os (Lonetto et al. 1992; Gruber and Gross 2003; Paget and Helmann 2003). Group $2 \sigma s$, most closely related to the Group $1 \sigma \mathrm{s}$, have $\sigma_{2-4}$ but are nonessential; Group $3 \sigma \mathrm{s}$ are less related but also contain $\sigma_{2-4}$; Group $4 \sigma \mathrm{s}$ (ECF $\sigma \mathrm{s}$ ) are the most minimal $\sigma \mathrm{s}$, containing only $\sigma_{2}$ and $\sigma_{4}$. Here, we examine Group 3 $\sigma s$. Most bacteria have multiple $\sigma s$ of this type, and Escherichia coli has two: $\sigma^{28}$ and $\sigma^{32}$ (Gruber and Gross 2003; Paget and Helmann 2003). $\sigma^{28}$, the most widely distributed alternative $\sigma$, controls flagella-related genes in all motile Gram-negative and Gram-positive bacteria and development in some nonmotile bacteria /Chilcott and Hughes 2000; Yu and Tan 2003; Serizawa et al. 2004; Shen et al. 2006). $\sigma^{32}$ controls the heat-shock response and is present in most proteobacteria (Nakahigashi et al. 1995; Guisbert et al. 2008). $\sigma^{32}$ and $\sigma^{28}$ each direct RNAP to a limited number of promoters $\left(\sim 50\right.$ for $\sigma^{32}$ and $\sim 25$ for $\sigma^{28}$ in E. coli) (Nonaka et al. 2006; Shen et al. 2006; Wade et al. 2006; Zhao et al. 2007). Expression from these promoters is instituted synchronously in response to a change in the amount and/or activity of the respective $\sigma$.
We previously found an important distinction between the promoter recognition properties of the E. coli Group 3 $\sigma s$ and their housekeeping $\sigma, \sigma^{70}$. In $\sigma^{70}$, the $-35,-10$, and E-10 motifs are partially redundant, and functional promoters are constructed from subsets of motifs (HookBarnard and Hinton 2007). Most commonly, promoters have -10 and -35 motifs but lack the E-10 (Lisser and Margalit 1993). Conversely, promoters with the E-10 motif do not have good matches to both the -10 and -35 motifs (Mitchell et al. 2003). Most E-10 promoters require neither the -35 motif nor its $\sigma$ recognition region (4.2) (Ponnambalam et al. 1986; Kumar et al. 1993; Minakhin and Severinov 2003; Young et al. 2004); a few have an excellent -35 and a reduced requirement for -10 (Thouvenot et al. 2004; Hook-Barnard et al. 2006). In sharp contrast, functional $\sigma^{28}$ and $\sigma^{32}$ promoters require good matches to the $-35,-10$, and E-10 motifs (Fig. 1B; Koo et al. 2009a,b). Consistent with this, the information content of the core regions of $\sigma^{32}$ promoters (18.3 bits) (Nonaka et al. 2006) and $\sigma^{28}$ promoters (21.3 bits) (determined from sequences of all E. coli $\sigma^{28}$ promoters listed in BioCyc [http://biocyc.org]; V Rhodius, unpubl.; data not shown) is much higher than that for $\sigma^{70}$ promoters (9.2 bits) (Shultzaberger et al. 2007). Requiring extensive recognition determinants would limit transcription to a relatively restricted set of promoters, making it important to understand how these $\sigma \mathrm{s}$ maintain dependence on all three promoter motifs.

We show that $\sigma^{32}$ and $\sigma^{28}$ require all three core promoter motifs for function largely because of altered residues in Region 2.3, a 17-amino-acid section of $\sigma_{2}$ implicated in promoter melting. Both $\sigma$ s deviate in sequence from critical Region 2.3 residues that are universally conserved in the housekeeping $\sigma$ s and are known to be important in melting. Converting these $\sigma^{32}$ and $\sigma^{28}$ residues to those present in the housekeeping $\sigma \mathrm{s}$ decreases the requirement for extensive recognition motifs and increases melting capacity at nonoptimal promoters. Our results suggest that extensive recognition motifs compensate for nonoptimal Regions 2.3 in $\sigma^{32}$ and $\sigma^{28}$. All Group 3 and Group $4 \sigma$ s have nonconsensus amino acids at the Region 2.3 positions we investigated. Decreasing melting capacity may be a general strategy enabling divergent alternative $\sigma$ s to mount a discrete, focused, and structured response to altered conditions.

\section{Results}

Phylogenetic analysis identifies elements required to bypass the $\sigma^{32}$ requirement for an E-10 motif

$\sigma^{32}$ s from different proteobacterial groups differ in their stringency of promoter recognition and in their recognition determinants. $\sigma^{32} \mathrm{~s}$ from E. coli and other $\gamma$-/ $\beta$-proteobacteria exhibit high dependence on the E-10 motif and recognize it with a universally conserved residue (K130) in $\sigma_{3}$ (Koo et al. 2009a). In contrast, $\alpha-/ \delta$-/E-proteobacteria show little to no dependence on the E-10 motif, and their comparable $\sigma_{3}$ residue is alanine, serine, or glutamine (Green and Donohue 2006; McGrath 
et al. 2007; Koo et al. 2009a). $\sigma^{32}$ of the $\alpha$-proteobacterium Caulobacter crescentus has little dependence on the E-10 motif even in combination with E. coli core RNAP (Koo et al. 2009a), indicating that the distinction in promoter recognition stringency resided within $\sigma^{32}$ itself. This raises the question of how C. crescentus $\sigma^{32}$ bypasses the requirement for the E-10 motif.

Comparison of E. coli and C. crescentus $\sigma^{32}$ revealed that the two differ in important residues in Region 2.3 (Fig. 2A), but not in residues implicated in base-specific recognition of promoters (Fig. 1C) or in Region 2.2, the most important core-binding motif (data not shown). Four Region 2.3 aromatic amino acids are universally conserved in housekeeping os (bold in Fig. 2A). Of these, F427

A

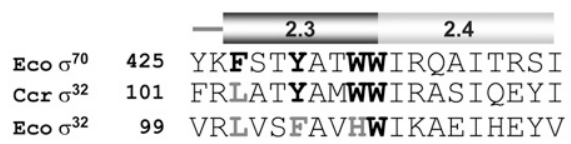

B

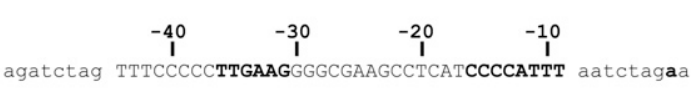

C

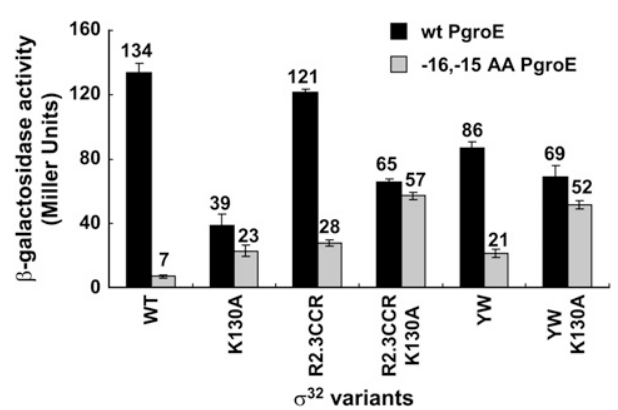

Figure 2. Identification of elements that bypass the need for the E-10 motif in $\sigma^{32}$. (A) Amino acid sequence alignment of a segment of Region 2.3 and Region 2.4 of E. coli $\sigma^{70}$, E. coli $\sigma^{32}$, and $C$. crescentus $\sigma^{32}$. Bold letters are residues universally conserved in housekeeping and Group $2 \sigma s$, and are implicated in promoter melting in $\sigma^{70}$; analogous residues that deviate from consensus in $\sigma^{32}$ s are in gray. The numbers indicate amino acid sequence position. $(B)$ Sequence of $\sigma^{32}$-dependent promoter (PgroE) used in this study. Only the wild-type sequence of groE promoter is shown; promoter variants were constructed from this sequence (Koo et al. 2009a). The native sequence of the groE promoter region is shown in capital letters, vector sequence is in lowercase, and -35 and -10 regions and transcription start site are shown in bold. (C) Expression from wild-type or $-16,-15 \mathrm{AA}$ mutant gro $E$ promoters by $E$. coli RNAP containing $\sigma^{32}$ variants. $\beta$-galactosidase activity (Miller units) of promoter::lac $Z$ fusions for each $\sigma^{32}$ variant on the wild-type promoter (black bar) or the $-16,-15$ AA mutant promoter lacking the extended -10 region (gray bar) are shown. These assays were performed in CAG57101 $(\Delta r p o H)$ with plasmids expressing $\sigma^{32}$ variants and bearing groE promoter variants::lac $Z$. For the $\beta$-galactosidase assays shown in this and subsequent figures, all values (indicated above each bar) are averages of at least three independent experiments; error bars indicate one standard deviation. Wild type (WT) is E. coli $\sigma^{32}$; variants have substitutions in E. coli $\sigma^{32}$ as follows: R2.3CCR, sequence encoding Region 2.3 is replaced with Region 2.3 of $C$. crescentus $\sigma^{32}$; R2.3CCR K130A is R2.3CCR with K130A; YW is $\mathrm{F} 104 \mathrm{YH} 107 \mathrm{Wr}^{32}$; and YW K130A is YW with K130A. is buried and is likely to play a structural role (Murakami et al. 2002), W434 is likely to be involved in promoter recognition (Juang and Helmann 1994), and Y430 and W433 are most directly involved in promoter DNA melting (Juang and Helmann 1994; Fenton et al. 2000; Tomsic et al. 2001). Three of these residues are present in C. crescentus $\sigma^{32}$ (and in $\sigma^{32}$ of all $\alpha$-proteobacteria), but only one is present in E. coli $\sigma^{32}$ (and in $\sigma^{32}$ of all other $\gamma$-proteobacteria) (Kourennaia and deHaseth 2007). We tested whether transplantation of $C$. crescentus Region 2.3 residues $\left({ }_{103}\right.$ LATYAMWW $\left._{110}\right)$ into $E$. coli $\sigma^{32}$ reduced dependence on the E-10 motif using lac $Z$ reporter assays driven from the $g r o E \sigma^{32}$ promoter (Fig. $2 \mathrm{~B}$ ) or derivatives of this promoter. Whereas authentic E. coli $\sigma^{32}$ exhibited a 20-fold dependence (Fig. 2C, columns 1,2), the hybrid $\sigma^{32}$ showed only a fourfold dependence (Fig. 2C, columns $5,6)$; introducing the K130A substitution to more fully mimic authentic $C$. crescentus $\sigma^{32}$ eliminated dependence on the E-10 motif (Fig. 2C, columns 7,8) and reduced the deleterious effect of the $\mathrm{K} 130 \mathrm{~A}$ substitution alone (Fig. 2C, cf. columns 7,8 and 3,4). Importantly, this effect is recapitulated by simply changing the residues in E. coli $\sigma^{32}$ analogous to Y430 and W433 to their counterparts in the housekeeping $\sigma \mathrm{s}$ and in C. crescentus $\sigma^{32}$ (creating F104YH107W ${ }^{32}$; YWo ${ }^{32}$ ) (Fig. 2C, cf. columns 9,10 and 5,6, and columns 11,12 , and 7,8 ). Note that $Y 430$ and $\mathrm{W} 433$ are the residues most directly implicated in promoter DNA melting (Juang and Helmann 1994; Fenton et al. 2000; Tomsic et al. 2001). Single amino acid substitutions showed a partial effect, and a triple mutant (L101F, F104Y, H107W) is virtually indistinguishable from $Y_{W} \sigma^{32}$ (data not shown). These results suggest that these YW residues in C. crescentus (and other $\alpha-/ \delta$ - $/ \varepsilon$-proteobacteria) enable their $\sigma^{32}$ s to bypass the requirement for an E-10 motif.

\section{$Y W \sigma^{32}$ generally suppresses defects of nonoptimal promoters}

We tested whether $\mathrm{YW \sigma}^{32}$ was generally proficient in transcribing nonconsensus promoters (Fig. 3). $\mathrm{YW}^{32}$ significantly suppressed the two -35 and the three -10 mutations in vivo: Whereas most mutations resulted in a fourfold to fivefold decrease in expression with wildtype $\sigma^{32}$, they showed only a 1.5 -fold to twofold decrease in expression with $\mathrm{YW \sigma}^{32}$ (Fig. 3A). Likewise, YWo ${ }^{32}$ showed reduced dependence on the length of the spacer DNA separating the -35 and -10 elements (Fig. 3B). This effect was reproduced in vitro. All mutant promoters with a significant transcription defect $(\leq 40 \%$ of the wildtype promoter) showed significantly higher transcription with $\mathrm{YW \sigma}^{32}$ than with wild-type $\sigma^{32}$, indicating that effects were direct (Fig. 3C,D). We conclude that $\mathrm{YW \sigma}^{32}$ generally exhibited higher activity than authentic $\sigma^{32}$ on weak promoters.

\section{Altering Region 2.3 in $\sigma^{28}$ also broadens promoter recognition}

$\sigma^{28}$ is highly divergent from $\sigma^{32}$ (Gruber and Gross 2003; Paget and Helmann 2003), enabling us to test whether the 
A

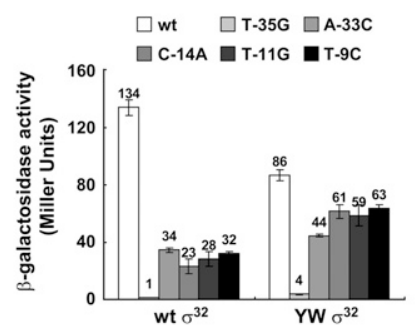

C

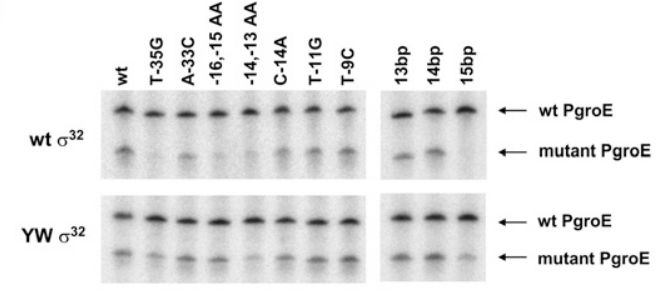

D

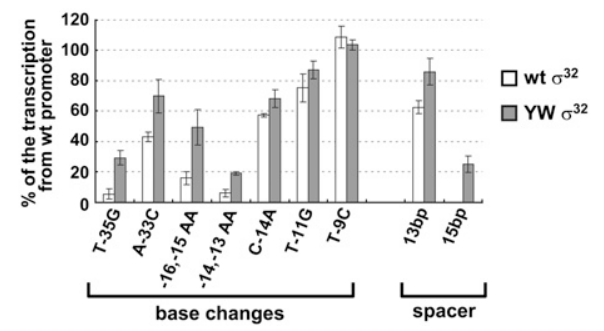

Figure 3. Suppression of nonoptimal promoter mutations by $\mathrm{YW}^{32}$ in vivo and in vitro. Expression from wild-type and mutant groE promoters $(A)$ or promoters with nonoptimal spacing between -10 and $-35(B)$ by wild-type and $\mathrm{YW} \sigma^{32}$ in vivo as assayed by $\beta$-galactosidase activities (Miller units) for each $\sigma^{32}$ variant. Assays were performed as described in Figure 2C. Numbers above each bar indicate average values of Miller units. $(C)$ Effect of YW substitution on transcription from nonoptimal groE promoter in vitro. Single-round run-off transcriptions were performed as described in the Materials and Methods; representative gels of each transcription reaction are shown. The top band in each lane is from the wild-type groE promoter (internal control); the bottom band originates from either the wild-type groE promoter or the mutant to be tested. $(D)$ Quantification of the in vitro transcripts. The bars indicate the relative transcription from mutant promoters as a percentage of transcript from wild-type promoter for each $\sigma^{32}$ variant. Each value was calculated as follows: (1) Each short transcript (lower band) was expressed as a percentage of the long transcript in the same lane to obtain the normalized short transcript; and (2) normalized short transcripts from each promoter variant (shown in $C$, lanes 2-11) were divided by normalized short transcript from the wild-type promoter (shown in $C$, lane 1). All values are averages of three independent experiments.

relationship between Region 2.3 consensus and increased transcription of weak promoters was likely to be generalizable to other Group 3 os. Comparison with $\sigma^{70}$ indicated that only two of the four aromatic Region 2.3 residues universally conserved in the housekeeping $\sigma \mathrm{s}$ are present in E. coli $\sigma^{28}$ (Fig. 4A). R74 (W434 in $\sigma^{70}$ ) was not considered further as it is highly conserved among $\sigma^{28}$ orthologs and participates in nonspecific promoter binding (Koo et al. 2009b). Q73 (W433 in $\sigma^{70}$ ) varies in different $\sigma^{28}$ s but is never W. We investigated whether $\mathrm{Q} 73 \mathrm{~W} \sigma^{28}$ has broadened promoter recognition.

$\sigma^{28}$ is exceptionally sensitive to substitution in the -10 region of the promoter, and the Q73W substitution dramatically increases the ability of $\sigma^{28}$ to recognize a variety of nonoptimal promoters. We examined promoter activity using lac $Z$ reporter assays driven from the tar $\sigma^{28}$ promoter (Fig. 4B) and its derivates. Eliminating the E-10 motif $(-14 G,-13 \mathrm{C})$ or altering any one of three bases in the -10 motif reduces expression by wild-type $\sigma^{28} \sim 30$-fold to 400-fold in vivo (Fig. 4C; Koo et al. 2009b). In sharp contrast, Q73W $\sigma^{28}$ exhibits only a threefold to 18-fold decrease on this same set of promoters (Fig. 4C). Importantly, Q73A does not increase expression from these mutant promoters, indicating that the suppressive effect resulted from adding the $\mathrm{W}$ residue at position 73 , rather than removing the naturally occurring $Q$ residue (data not shown). Likewise, Q73W exhibits 10-fold suppression of the transcription defects resulting from removing each of the three $\sigma^{28}$ residues known to participate in base-specific recognition of the $\sigma^{28}$ promoter (R91 [E-10 motif] and D81/R84 [-10 motif]) (Fig. 4D; Koo et al. $2009 \mathrm{~b}$ ) and shows enhanced tolerance for variation in the length of the spacer DNA separating the -35 and -10 elements (Fig. 4E). All promoters with significant transcriptional defects in vitro $\leq 40 \%$ of the wild-type promoter) showed significantly increased transcription with Q73W $\sigma^{28}$ as compared with wild-type $\sigma^{28}$ (Fig. 4F). Thus, $\mathrm{Q} 73 \mathrm{~W} \sigma^{28}$ is directly responsible for significantly enhanced transcription of a broad range of nonoptimal promoters.

\section{$Y W \sigma^{32}$ broadens promoter recognition of natural promoters}

Thus far, we examined the effects of $Y W \sigma^{32}$ on a promoter set, each differing from the groE consensus promoter at only a single position. Natural promoters may have many changes from the consensus and an UP-element (Zhao et al. 2005; Nonaka et al. 2006; Wade et al. 2006), recognized by the C-terminal domain of the $\alpha$-subunit (Ross et al. 1993; Gourse et al. 2000), which might obscure the effect of $\mathrm{YW \sigma}^{32}$ on natural promoters. We compared expression of natural $\sigma^{32}$ promoters in $E$. coli fused to a green fluorescent protein (GFP) reporter driven by either wild-type $\sigma^{32}$ or $\mathrm{YW}^{32}$ (see the Materials and Methods). The promoter library encompassed sequences -65 to +20 relative to the start site of transcription, and therefore included any UP-element present. We found that promoters very weakly transcribed by $\sigma^{32}$ RNAP were more strongly transcribed by $\mathrm{YW} \sigma^{32} \mathrm{RNAP}$; conversely, promoters strongly transcribed by $\sigma^{32}$ RNAP have similar or less expression with $Y W \sigma^{32}$ RNAP. This is illustrated by comparing the ratio of promoter strengths by $\sigma^{32}$ or YW $\sigma^{32}$ RNAP against the strength of the promoter in the presence of $\sigma^{32}$ RNAP (Fig. 5A; see also Supplemental Fig. S2 for the raw data). Therefore, $\mathrm{YW} \sigma^{32}$ preferentially increases expression from weaker, less conserved natural promoters. We consider the implications of this finding in the Discussion. 
Koo et al.

A

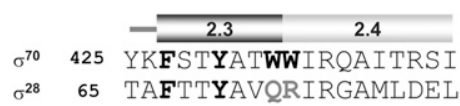

B

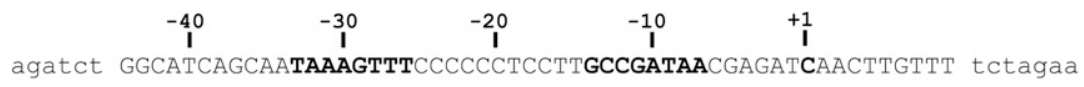

C

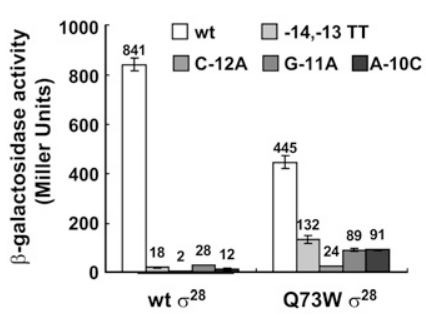

$\mathbf{F}$

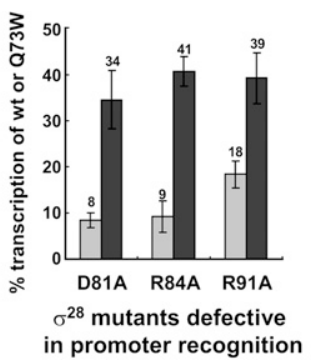

D

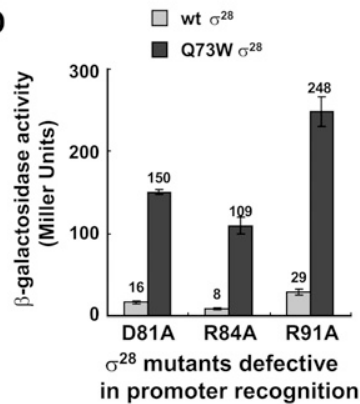

E

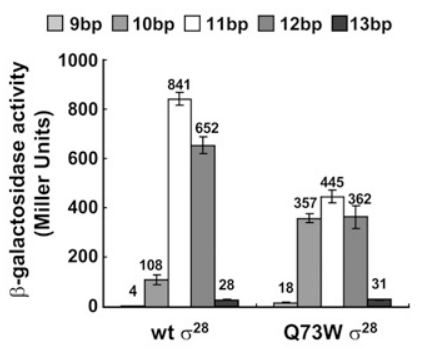

Figure 4. Enhanced promoter utilization by Q73W $\sigma^{28}$ in vivo and in vitro. (A) Amino acid sequence alignment of Regions 2.3 and 2.4 of $E$. coli $\sigma^{70}$ and $\sigma^{28}$. Bold letters are residues implicated in promoter melting in $\sigma^{70}$, and the residues not conserved in $\sigma^{28}$ are in gray. The numbers indicate amino acid sequence position. $(B)$ Sequence of $\sigma^{28}$-dependent promoter (Ptar) used in this study. Only the wildtype sequence of the tar promoter is shown; promoter variants were constructed from this sequence (Koo et al. 2009b). The native sequence of the tar promoter region is shown in capital letters, vector sequence is in lowercase, and -35 and -10 regions and transcription start site are shown in bold. $(C)$ Expression from tar promoter mutants by RNAP containing wild-type or Q73W $\sigma^{28}$ in vivo. $\beta$-Galactosidase activities (Miller units) of promoter::lac $Z$ fusions are shown. Assays were performed in CAG57115 ( $\Delta$ fliA, $\Delta$ flgM) with plasmids expressing $\sigma^{28}$ variants and bearing tar promoter variants::1acZ. All values are averages of three independent experiments. $(D)$ In vivo effects of the Q73W substitution on mutations in $\sigma^{28}$ that eliminate promoter recognition determinants. $\beta$-Galactosidase activities (Miller units) driven by each $\sigma^{28}$ variant on tested promoters are shown. All values are averages of three independent experiments. (Gray bar) Activity of each mutation in wild-type background; (black bar) activity of each mutation in Q73W background. (E) Expression from tar promoter mutants with nonoptimal spacers by RNAP containing wild-type or Q73W $\sigma^{28}$ in vivo. $\beta$-Galactosidase activities (Miller units) are shown. All values are averages of three independent experiments. The spacer length was varied from 9 to 13 base pairs $(\mathrm{bp})$; the wild-type tar promoter has an 11-bp spacer. $(F)$ Effects of the Q73W substitution on mutations in $\sigma^{28}$ that eliminate promoter recognition determinants and of tar promoter variants in vitro. Relative transcription determined from single-round run-off transcription assays (Supplemental Fig. S1) is depicted as bar graphs. Each experiment was repeated a minimum of three times, and numbers above each bar indicate average values of relative transcription. (Left graph) Effect of Q73W substitution on mutations eliminating promoter recognition determinants in $\sigma^{28}$. Percent transcription was calculated as (intensity of transcript for RNAP with wild-type or Q73W $\sigma^{28}$ carrying the specified additional substitutions in $\sigma^{28}$ )/(intensity of transcript for RNAP with wildtype or Q73W $\sigma^{28}$ ). (Middle and right graphs) Expression from tar promoters with base substitutions (middle) and nonoptimal spacer lengths (right) by RNAP containing wild-type or Q73W $\sigma^{28}$, normalized as described in Figure 3D.

\section{Altered Region 2.3 residues exert their effects on a step beyond duplex promoter binding}

The $\sigma^{70}$ residues analogous to those altered in $\sigma^{28}$ and/or $\sigma^{32}$ (Y430 and W433) have a minor effect on initial recognition of duplex DNA and a major effect on open complex formation (Fenton et al. 2000; Tomsic et al. 2001; Schroeder et al. 2009). The crystal structure of Aquifex aeolicus $\sigma^{28}$ complexed to its anti- $\sigma$ indicates that these Region 2.3 residues in Group $3 \sigma$ s are roughly in the same position as in the housekeeping $\sigma$ s with respect to the most C-terminal helix of $\sigma_{2}$ (Sorenson et al. 2004), suggesting that they might play similar roles in both $\sigma$ families. We examined whether aromatic amino acid-substituted $\sigma^{32}$ and $\sigma^{28}$ holoenzymes $\left(E \sigma^{32}\right.$ and $\left.E \sigma^{28}\right)$ were altered in initial duplex DNA recognition or in open complex formation using assays developed to characterize $\sigma^{70}$ holoenzyme $\left(E \sigma^{70}\right)$. Consistent with their effects in $\mathrm{E} \sigma^{70}$, these residues have little or no effect on duplex binding by $\mathrm{E} \sigma^{32}$ and $\mathrm{E} \sigma^{28}$ (Fig. 6A,B) and exert their major effects on strand opening (Fig. 6C,D). 
A

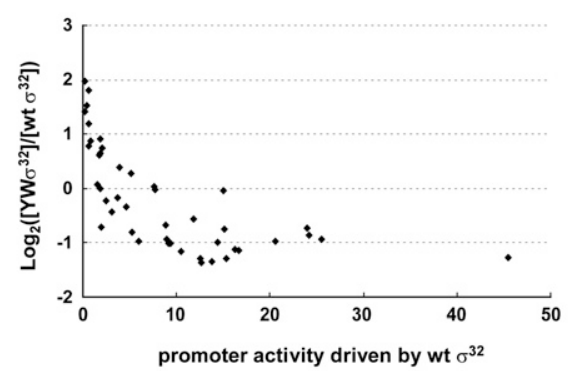

B

Information content (bits) of aligned promoter motifs
\begin{tabular}{|c|ccccc|}
\hline Promoters & \multicolumn{5}{|c|}{ Promoter motifs } \\
more active with & UP & -35 & spacer & E-10 & -10 \\
\hline wt $\sigma^{32}$ & 3.5 & 8.3 & 2.1 & 2.2 & 6.3 \\
YW $\sigma^{32}$ & 4.6 & 6.0 & 2.4 & 2.5 & 5.9 \\
\hline
\end{tabular}

Figure 5. $\mathrm{YW \sigma}^{32}$ preferentially increases expression of weak $\sigma^{32}$ promoters in vivo. Promoter activities driven by either wild type $(w t)$ or $Y W \sigma^{32}$ were determined by the expression of GFP from $50 \sigma^{32}$ promoters (Nonaka et al. 2006) as described in the Materials and Methods. (A) Data are displayed as a scatter plot showing the $\log 2$ value of the ratio of promoter activities driven by $Y W \sigma^{32}$ and wild-type $\sigma^{32}$ (Y-axis) versus the strength of the promoter when driven by wild-type $\sigma^{32}$ ( $X$-axis). Promoter activity was calculated from the slope of the differential plot of $\mathrm{OD}_{600}$ versus GFP fluorescence (RFU); see the Materials and Methods. (B) Information content of promoters more active with either wild type (30 promoters) or YW $\sigma^{32}$ (19 promoters). Values were calculated as described in the Materials and Methods. Each motif covers the following sequences: UP element, -60 to -45 ; -35 motif, -37 to -31 ; spacer between -35 and -10 motif; extended -10 motif, -16 and -15 ; and -10 motif, -14 to -9 .

We assessed initial recognition by determining $E \sigma^{32}$ and $\mathrm{E} \sigma^{28}$ binding to duplex DNA at $4^{\circ} \mathrm{C}$, a temperature that prevents strand opening and traps the initial rapidly dissociating complex between RNAP and DNA, as shown for $\mathrm{E}^{70}$ (Fenton et al. 2000). The low-temperature complex is on the pathway to the open complex, and therefore provides an accurate estimate of initial binding ( $\mathrm{Li}$ and McClure 1998). These trapped complexes are expected to be sensitive to inhibition by heparin, which binds free RNAP irreversibly, thereby inactivating rapidly dissociating RNAP. We assessed binding both to the complete promoter (consensus -35 , E-10, and -10 elements) and to a suboptimal promoter (lacking the E-10 motif). YW $\sigma^{32}$ holoenzyme $\left(\mathrm{E}-\mathrm{YW} \sigma^{32}\right)$ and wild-type $\sigma^{32}$ holoenzyme $\left(\mathrm{E}-\mathrm{wt} \sigma^{32}\right)$ show equivalent extents of heparin-sensitive binding (Fig. 6A), providing clear evidence that $\mathrm{YW \sigma}^{32}$ does not affect initial binding. Likewise, $\mathrm{Q} 73 \mathrm{~W} \sigma^{28}$ holoenzyme $\left(\mathrm{E}-\mathrm{Q} 73 \mathrm{~W} \sigma^{28}\right)$ and wild-type $\sigma^{28}$ holoenzyme $\left(\mathrm{E}-\mathrm{w} \mathrm{t \sigma}^{28}\right)$ show equivalent extents of binding (Fig. 6B). This binding is partially heparin-resistant, possibly reflecting formation of an "intermediate" complex further down the pathway. In any case, E-Q73W $\sigma^{28}$ and $\mathrm{E}-\mathrm{w} t \sigma^{28}$ do not display any distinction in behavior in this assay, consistent with the idea that initial steps in the process are unaffected. Very similar results were obtained for $E \sigma^{32}$ and $E \sigma^{28}$ using shorter templates truncated just downstream from the -10 regions of each promoter (data not shown). Additionally, we validated that the observed binding of $E \sigma^{28}$ and $E \sigma^{32}$, although weak, is dependent on specific promoter sequences, as it is not observed with random sequence DNA (Supplemental Fig. S3). Taken together, these results support the conclusion that the Region 2.3 alterations in $\sigma^{28}$ and $\sigma^{32}$ have a minimal effect on duplex recognition.

To examine strand opening, we assessed heparinresistant binding to "fork junction" templates at $4^{\circ} \mathrm{C}$. A fork junction template is one in which the template strand is truncated just upstream of the position of strand opening (T-11 for $\mathrm{P}_{\text {groE }}$ with $\mathrm{E \sigma}^{32}$ [Mecsas et al. 1991] and T-9 for $\mathrm{P}_{\text {tar }}$ with $\mathrm{E \sigma}^{28}$ [Givens et al. 2001]), and the nontemplate strand continues as a single-strand overhang. This assay has been validated both kinetically and structurally to be an excellent mimic of open complex formation in $\mathrm{E}^{70}$ (Guo and Gralla 1998; Murakami et al. 2002; Tsujikawa et al. 2002). Indeed, the extent to which a particular combination of fork junction template and holoenzyme is able to form a stable complex (i.e., resistant to heparin challenge) reflects the propensity for open complex formation with the particular set of reactants used (Guo and Gralla 1998; Fenton et al. 2000; Tsujikawa et al. 2002). We observe clear evidence that both $\mathrm{YW} \sigma^{32}$ and $\mathrm{Q} 73 \mathrm{~W} \sigma^{28}$ are more proficient than their wild-type counterparts at promoting formation of the open complex, when assayed with the appropriate templates. E-YWo ${ }^{32}$ shows fivefold enhancement of open complex formation when assayed on a suboptimal template (no E-10) whose fork extends to $-9\left(8 \%\right.$ E-wt $\sigma^{32}$ vs. $41 \% \mathrm{E} \mathrm{YW} \sigma^{32}$ ) (Fig. 6C, $\left.-16,-15 \mathrm{AA}\right)$. The other templates are almost completely shifted by E-wto ${ }^{32}$ and therefore cannot provide distinction between the two holoenzymes. The effect is even more dramatic for $\sigma^{28}$, where E-Q73W $\sigma^{28}$ exhibits $\geq 20$-fold more open complex formation than E-wto ${ }^{28}$ at both the long and short suboptimal fork junction templates (Fig. 6D; $-14,-13$ AA). We also validated that the observed binding of $\mathrm{E} \sigma^{28}$ and $\mathrm{E} \sigma^{32}$ to fork junction templates is dependent on specific promoter sequences, as no specific binding to fork junction templates bearing their anti--10 promoter sequences was observed (Supplemental Fig. S3). Taken together, these results provide strong support for the idea that $\mathrm{YW \sigma}^{32}$ and $\mathrm{Q} 73 \mathrm{~W} \sigma^{28}$ significantly promote open complex formation at suboptimal promoter templates.

\section{Discussion}

Early "primordial" $\sigma$ factors have diverged into major subgroups: the housekeeping $\sigma \mathrm{s}$ such as $\sigma^{70}$, and the alternative $\sigma \mathrm{s}$. This divergence spawned evolution of an important gene expression strategy, allowing differentially regulated $\sigma s$ to recognize discrete classes of promoters. $\sigma$ factor specialization has another distinguishing feature: Housekeeping $\sigma$ s recognize a large number of diverse promoters $(>1000)$, whereas most alternative $\sigma$ s are much more restrictive in promoter selection, with a tighter requirement for the sequence and spacing of 
Koo et al.

A

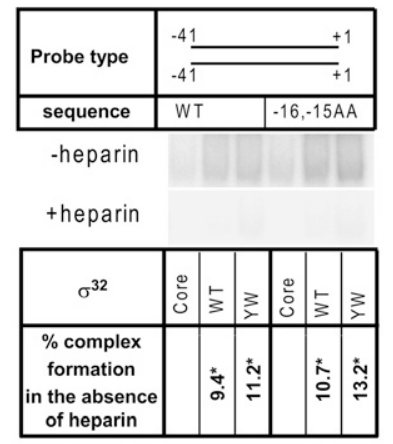

C

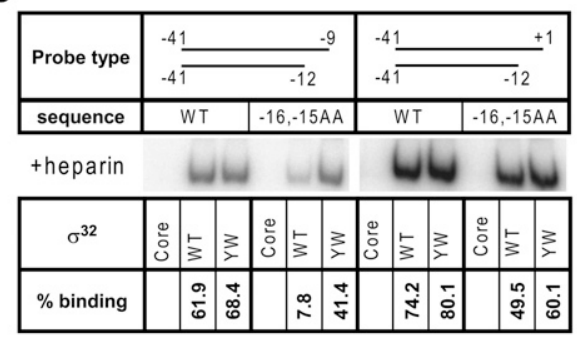

B

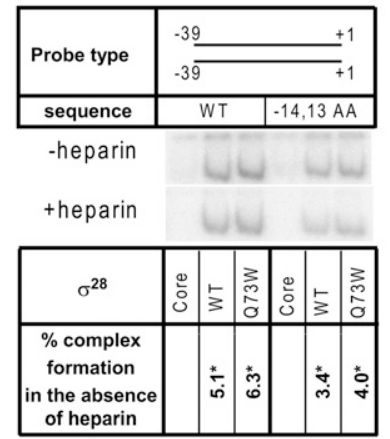

D

\begin{tabular}{|c|c|c|c|c|c|c|c|c|c|c|c|c|}
\hline \multirow{2}{*}{ Probe type } & \multicolumn{6}{|c|}{-39} & \multicolumn{3}{|c|}{-39} & & \multicolumn{2}{|c|}{+1} \\
\hline & \multicolumn{3}{|c|}{-39} & \multicolumn{3}{|c|}{-10} & \multicolumn{3}{|c|}{-39} & \multicolumn{3}{|c|}{-10} \\
\hline sequence & \multicolumn{3}{|c|}{ WT } & \multicolumn{3}{|c|}{$-14,-13 \mathrm{AA}$} & \multicolumn{3}{|c|}{ WT } & \multicolumn{3}{|c|}{$-14,-13 \mathrm{AA}$} \\
\hline +heparin & & 6 & & & & 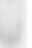 & & $\mathbf{y}$ & $\omega$ & & & $\omega$ \\
\hline$\sigma^{28}$ & $\begin{array}{l}0 \\
0 \\
0\end{array}$ & 々 & 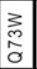 & 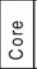 & $\stackrel{5}{3}$ & 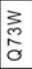 & 凹ั & 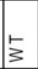 & 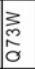 & ¿ั0 & 15 & $\begin{array}{l}3 \\
\text { â } \\
\text { ô }\end{array}$ \\
\hline$\%$ binding & & $\stackrel{m}{\sigma}$ & $\stackrel{\text { O̊ }}{\stackrel{0}{0}}$ & & $\delta_{0}$ & $\underset{\text { mp }}{\text { p. }}$ & & $\frac{4}{i n}$ & กั่ & & $\stackrel{\leftrightarrow}{\longrightarrow}$ & ब্ল \\
\hline
\end{tabular}

Figure 6. The altered Region 2.3 residues in $\sigma^{32}$ and $\sigma^{28}$ affect open complex formation but not template binding. Electrophoretic mobility shift assay was performed as described in the Materials and Methods (also contains sequences of the DNA probes). End-labeled probe $(10 \mathrm{nM})$ and holoenzyme $(25 \mathrm{nM})$ (both as shown in the figure) were incubated for 10 min at $4^{\circ} \mathrm{C}$. In heparin challenge experiments, $100 \mu \mathrm{g} / \mathrm{mL}$ heparin was added and incubation was continued for $5 \mathrm{~min}$. All values shown are averages of at least three independent experiments. Standard deviations are $\leq 25 \%$ of average values. Representative gels are shown. $(A, B)$ Binding to duplex DNA by E-wt $\sigma^{32}$ or E-YW $\sigma^{32}$ at the $g r o E$ promoter $(A)$ or by E-wt $\sigma^{28}$ or E-Q73W $\sigma^{28}$ at the tar promoter $(B)$. Holoenzyme-DNA complexes formed without (top) or with (bottom) heparin challenge are shown. The percent complex formation in the absence of heparin is considered to reflect initial duplex binding. $(C, D)$ Binding of RNAP to fork junction templates by E-wt $\sigma^{32}$ or E-YW $\sigma^{32}$ at the groE promoter $(C)$ or by $\mathrm{E}-\mathrm{wt} \sigma^{28}$ or E- Q73W $\sigma^{28}$ to the tar promoter $(D)$. Percent binding is the extent of formation of heparin-resistant complexes.

their promoter motifs. In this study, we asked what feature(s) of $\sigma s$ is responsible for this important difference in promoter recognition strategy. Our results suggest the hypothesis that the balance of melting and recognition functions of $\sigma \mathrm{s}$ is critical to setting the stringency of promoter recognition.

We investigated the features of $\sigma$ s required for stringent promoter recognition by $\sigma^{32}$ and $\sigma^{28}$, two highly divergent members of the Group $3 \sigma$ subfamily present in E. coli. Sequence variation in Region 2.3 is largely responsible for this requirement: Converting one or two residues in Region 2.3 to their counterpart(s) in the housekeeping os largely alleviated the stringent requirements. Our results indicated that the consensus variants were fivefold to $\geq 20$-fold more proficient than their wild-type $\sigma$ counterparts at promoting open complex formation on suboptimal promoters (Fig. 6C,D). Thus, E $\sigma^{32}$ and $E \sigma^{28}$ require highly specified promoters because of their reduced capacity for promoter melting.

os perform two sequential activities: recognizing promoter motifs (to position RNAP), and facilitating subsequent steps required for melting the -10 region to create the "open complex" poised for transcription initiation. These activities are tightly coupled: The Region 2.3 residues facilitating melting and general duplex recognition are located in the same $\alpha$-helix as the Region 2.4 residues that recognize specific bases in the -10 region and then stabilize the melted state by interaction with the nontemplate strand. In $E \sigma^{70}$ promoters, strand separation is nucleated within the -10 recognition motif, most likely by flipping out the $-11 \mathrm{~A}$ base (Lim et al. 2001; Schroeder et al. 2009). W433 may "push" the -11A out of the helix (Tomsic et al. 2001). Y430 is believed to interact with and stabilize the conformation of the flipped out $-11 \mathrm{~A}$ (Schroeder et al. 2009). Importantly, it is altering $\sigma^{32}$ and $\sigma^{28}$ residues at positions analogous to Y430 and W433 to those in housekeeping $\sigma \mathrm{s}$ $\left(\mathrm{F} 104 \mathrm{YH} 107 \mathrm{Wr}^{32} ; \mathrm{Q} 73 \mathrm{W \sigma}^{28}\right)$ that increases melting and relaxes promoter recognition.

How might a completely consensus promoter decrease the requirements for $\sigma$ melting functions performed by Y430 and W433? The kinetics of open complex formation by E. coli $\sigma^{70}$ holoenzyme at a consensus promoter (consensus $-35, \mathrm{E}-10,-10$ ) provide a way of thinking about this linkage (Schroeder et al. 2009). It is suggested that the completely consensus promoter greatly stabilizes the transition state of the normally rate-limiting step in open complex formation so that it becomes a kinetically significant intermediate, possibly through contacts between the E-10 region and RNAP. The implication is that base flipping and strand separation, previously coupled kinetically, become two separable steps 
at the consensus promoter, with the latter being ratelimiting. Thus, the defect in base flipping caused by a suboptimal amino acid sequence in $\sigma$ Region 2.3 would have no or little effect at consensus promoters but would lead to much reduced expression in weaker promoters. Consistent with this interpretation, Y430 and W433 substitutions do not affect open complex formation at the consensus $\sigma^{70}$ promoter, although they have big effects on melting at standard promoters (Tomsic et al. 2001; Schroeder et al. 2009). Thus, the conformational changes driven by the consensus promoter elements obviate the requirement for these Region 2.3 residues. This scenario precisely explains our findings for $\sigma^{32}$ and $\sigma^{28}$ : Their consensus promoters drive melting even though substitutions in residues analogous to Y430 and/ or W433 reduce the melting proficiency of $\sigma^{32}$ and $\sigma^{28}$.

The suboptimal melting capacity of $\sigma^{32}$ and $\sigma^{28}$ has biological correlates. The necessity of optimal placement and sequence of promoter motifs to create a functional promoter means that deviation from consensus has profound negative effects on promoter activity. This focuses transcription by these $\sigma$ s on their authentic regulons and decreases the possibility that their responses will be diminished because they engage in adventitious transcription of near-match promoters. Likewise, the exquisite sensitivity of transcriptional capacity to promoter sequence also allows promoter strength to be regulated over a broad range so that regulon members are produced in optimal amounts relative to each other. Finally, suboptimal melting allows these $\sigma$ s to maintain the integrity of their recognition across many organisms. $\sigma^{28}$ directs synthesis of flagellar components in both Gram-negative and Gram-positive bacteria, separated in evolution by billions of years. Yet the consensus sequence of the promoters recognized by $\sigma^{28}$ remains unchanged.

Our results graphically illustrate the extent to which the relative promoter strength is deregulated when the $\sigma$ factor has consensus melting determinants. Whereas the natural promoters in the $\sigma^{32}$ regulon members display an $\sim 100$-fold range in activity when driven by wild-type $\sigma^{32}$, only a 20 -fold range is seen when they are transcribed by the melting-proficient $\mathrm{YW}^{32}$, with the weakest promoters having enhanced activity and the strongest promoters showing decreased transcription by $\mathrm{YW \sigma}^{32}$ (possibly because of difficulty in promoter clearance) (Fig. 5A; Supplemental Fig. S2). Interestingly, motif comparison indicates that the predominant difference between promoters preferentially transcribed by $\mathrm{YW}^{32}$ and by wildtype $\sigma^{32}$ is that the former has a less conserved -35 motif (Fig. 5B). This distinction raises the intriguing possibility that wild-type $\sigma^{32}$, but not $\mathrm{YW} \sigma^{32}$, uses the -35 region as a "gatekeeper" both to determine functional promoters and to set promoter strength. A consensus -35 region may be necessary to slow dissociation sufficiently to permit strand opening.

It is interesting to consider why the $\sigma^{32} \mathrm{~s}$ of $\alpha$-protobacteria might have broadened promoter specificity. Interestingly, $\sigma^{32} \mathrm{~s}$ in the $\alpha$-protobacteria often control processes in addition to heat shock, such as development in Myxococcus xanthus (Ueki and Inouye 2001) or other stress responses (e.g., heavy metal stress in C. crescentus) (McGrath et al. 2007). Additionally, groEL, the most important member of the $\sigma^{32}$ regulon (Kusukawa and Yura 1988), is regulated by an alternative mechanism in $\alpha$-proteobacteria (Yura and Nakahigashi 1999). Thus, $\alpha$-proteobacterial $\sigma^{32}$ s may transcribe more genes without the necessity of finely controlling the extent of their expression as compared with $\gamma$-proteobacterial $\sigma^{32}$ s.

Ever since Helmann's seminal work (Juang and Helmann 1994) demonstrated the involvement of $\sigma$ Region 2.3 residues in promoter DNA melting, much effort has been devoted to defining its mechanism (Juang and Helmann 1994; Fenton et al. 2000; Schroeder et al. 2009). However, there has been little consideration of whether $\sigma$ melting proficiency differs among $\sigma$ subfamilies and whether this property is used to set the promoter recognition promiscuity of that family. Given that Group 3 os rarely use $>100$ promoters in any bacterial species (in marked contrast to the housekeeping $\sigma^{70}$ ) and that all of them share nonconsensus residues in Region 2.3 (Supplemental Fig. S4), we propose the hypothesis that amino acid sequence differences within Region 2.3 are important for the differences in breadth of promoter choice. Importantly, divergent Group $4 \sigma \mathrm{s}$ are also discrepant from the housekeeping $\sigma \mathrm{s}$ in some of these important Region 2.3 residues. Melting deficiencies in the more divergent alternative $\sigma s$ may be a universal mechanism to ensure their promoter recognition stringency.

\section{Materials and methods}

Details of materials and methods are presented in the Supplemental Material.

\section{Strains, plasmids, and growth conditions}

Strains and plasmids used in this study are listed in Supplemental Table S1. Cells were grown at $30^{\circ} \mathrm{C}$ in Luria-Bertani (LB) media supplemented with appropriate antibiotics such as ampicillin $(100 \mu \mathrm{g} / \mathrm{mL})$, chloramphenicol $(30 \mu \mathrm{g} / \mathrm{mL})$, kanamycin $(20$ $\mu \mathrm{g} / \mathrm{mL})$, and spectinomycin $(50 \mu \mathrm{g} / \mathrm{mL})$. For the strains lacking rpoH (CAG57101), cells were grown with $0.1 \% \mathrm{~L}-(+)$-arabinose to induce expression of GroESL (Koo et al. 2009a).

\section{$\beta$-galactosidase assay, purification of $\sigma s$, and in vitro} transcription

$\beta$-galactosidase assays (used to measure in vivo promoter::lacZ activities), overproduction and purification of $\sigma s$, and in vitro single-round transcription assays were performed essentially as described (Koo et al. 2009a,b). Details are in the Supplemental Material.

\section{Promoter activity determined by expression of GFP}

The $\sigma^{32}$ promoter library was constructed as described previously (Rhodius et al. 2006). Fifty $\sigma^{32}$ promoters validated in our previous work (Nonaka et al. 2006) were cloned as XholBamHI fragments into the GFP reporter plasmid, pUA66. Reporter strains were constructed by transforming derivatives of pSAKT32 and promoter library plasmids into CAG57101 sequentially using electroporation. 
Promoter assays were performed by direct inoculation of LB broth supplemented with appropriate antibiotics from fresh transformants. Fluorescence and $\mathrm{OD}_{600}$ were measured in a Varioskan spectrofluorometer (Thermo Electron Corporation). $\sigma^{32}$-dependent promoter activity was determined as described in the Supplemental Material.

\section{Calculation of information content of promoter motifs}

The information content $\left(I_{\text {seq }}\right)$ of aligned promoter motifs was calculated using

$$
I_{\text {seq }}=\sum_{i} \sum_{b} f_{b, i} \log _{2} \frac{f_{b, i}}{p_{b}},
$$

where $i$ is the position within the site, $b$ refers to each of the possible bases, $f_{b, i}$ is the observed frequency of each base at that position, and $p_{b}$ is the frequency of base $b$ in the entire genome (in E. coli, taken to be 0.25 for A/G/C/T) (Schneider et al. 1986).

\section{Electrophoretic mobility shift assay}

PAGE-purified synthetic oligonucleotides were used for preparing double-strand and fork junction probes. ${ }^{32}$ P-labeled $10 \mathrm{nM}$ annealed DNA probe and $25 \mathrm{nM}$ holoenzyme were mixed in 10 $\mu \mathrm{L}$ of binding buffer and incubated for $10 \mathrm{~min}$ at $4^{\circ} \mathrm{C}$. For heparin challenge, $2 \mu \mathrm{L}$ of $600 \mu \mathrm{g} / \mathrm{mL}$ heparin were added, and the incubation was continued for an additional $5 \mathrm{~min}$. Electrophoresis was performed in a prechilled $5 \%$ acrylamide/TBE gel at $4^{\circ} \mathrm{C}$. See details in the Supplemental Material.

\section{Acknowledgments}

We thank Tania Baker for her critical input in conceptualizing this work, Vivek Mutalik for technical assistance, and members of the Gross laboratory for useful comments. This work was supported by National Institutes of Health Grants GM057755 (to C.A.G.) and GM31808 (to P.L.dH.).

\section{References}

Amaya E, Khvorova A, Piggot PJ. 2001. Analysis of promoter recognition in vivo directed by $\sigma^{\mathrm{F}}$ of Bacillus subtilis by using random-sequence oligonucleotides. I Bacteriol 183: 3623-3630.

Asayama M, Imamura S. 2008. Stringent promoter recognition and autoregulation by the group $3 \sigma$-factor SigF in the cyanobacterium Synechocystis sp. strain PCC 6803. Nucleic Acids Res 36: 5297-5305.

Barne KA, Bown JA, Busby SJ, Minchin SD. 1997. Region 2.5 of the Escherichia coli RNA polymerase $\sigma^{70}$ subunit is responsible for the recognition of the 'extended-10' motif at promoters. $E M B O$ J 16: 4034-4040.

Britton RA, Eichenberger P, Gonzalez-Pastor JE, Fawcett P, Monson R, Losick R, Grossman AD. 2002. Genome-wide analysis of the stationary-phase $\sigma$ factor $\left(\sigma^{\mathrm{H}}\right)$ regulon of Bacillus subtilis. J Bacteriol 184: 4881-4890.

Browning DF, Busby SJ. 2004. The regulation of bacterial transcription initiation. Nat Rev Microbiol 2: 57-65.

Chilcott GS, Hughes KT. 2000. Coupling of flagellar gene expression to flagellar assembly in Salmonella enterica serovar typhimurium and Escherichia coli. Microbiol Mol Biol Rev 64: 694-708.

Daniels D, Zuber P, Losick R. 1990. Two amino acids in an RNA polymerase $\sigma$ factor involved in the recognition of adjacent base pairs in the -10 region of a cognate promoter. Proc Natl Acad Sci 87: 8075-8079.
Dombroski AJ, Walter WA, Record MT Jr, Siegele DA, Gross CA. 1992. Polypeptides containing highly conserved regions of transcription initiation factor $\sigma^{70}$ exhibit specificity of binding to promoter DNA. Cell 70: 501-512.

Dombroski AJ, Walter WA, Gross CA. 1993. Amino-terminal amino acids modulate $\sigma$-factor DNA-binding activity. Genes \& Dev 7: 2446-2455.

Eichenberger P, Jensen ST, Conlon EM, van Ooij C, Silvaggi J, Gonzalez-Pastor JE, Fujita M, Ben-Yehuda S, Stragier P, Liu JS, et al. 2003. The $\sigma^{\mathrm{E}}$ regulon and the identification of additional sporulation genes in Bacillus subtilis. I Mol Biol 327: 945-972.

Feklistov A, Barinova N, Sevostyanova A, Heyduk E, Bass I, Vvedenskaya I, Kuznedelov K, Merkiene E, Stavrovskaya E, Klimasauskas S, et al. 2006. A basal promoter element recognized by free RNA polymerase $\sigma$ subunit determines promoter recognition by RNA polymerase holoenzyme. Mol Cell 23: 97-107.

Fenton MS, Lee SJ, Gralla JD. 2000. Escherichia coli promoter opening and -10 recognition: Mutational analysis of $\sigma^{70}$. EMBO J 19: 1130-1137.

Gama-Castro S, Jimenez-Jacinto V, Peralta-Gil M, Santos-Zavaleta A, Penaloza-Spinola MI, Contreras-Moreira B, Segura-Salazar J, Muniz-Rascado L, Martinez-Flores I, Salgado H, et al. 2008. RegulonDB (version 6.0): Gene regulation model of Escherichia coli K-12 beyond transcription, active (experimental) annotated promoters and Textpresso navigation. Nucleic Acids Res 36: D120-D124. doi: 10.1093/nar/gkm994.

Gardella T, Moyle H, Susskind MM. 1989. A mutant Escherichia coli $\sigma^{70}$ subunit of RNA polymerase with altered promoter specificity. J Mol Biol 206: 579-590.

Givens JR, McGovern CL, Dombroski AJ. 2001. Formation of intermediate transcription initiation complexes at pfliD and pflgM by $\sigma^{28}$ RNA polymerase. J Bacteriol 183: 6244-6252.

Gourse RL, Ross W, Gaal T. 2000. UPs and downs in bacterial transcription initiation: The role of the $\alpha$ subunit of RNA polymerase in promoter recognition. Mol Microbiol 37: 687695.

Green HA, Donohue TJ. 2006. Activity of Rhodobacter sphaeroides RpoHII, a second member of the heat shock $\sigma$ factor family. J Bacteriol 188: 5712-5721.

Gruber TM, Gross CA. 2003. Multiple $\sigma$ subunits and the partitioning of bacterial transcription space. Annu Rev Microbiol 57: 441-466.

Guisbert E, Yura T, Rhodius VA, Gross CA. 2008. Convergence of molecular, modeling, and systems approaches for an understanding of the Escherichia coli heat shock response. Microbiol Mol Biol Rev 72: 545-554.

Guo Y, Gralla JD. 1998. Promoter opening via a DNA fork junction binding activity. Proc Natl Acad Sci 95: 1165511660.

Haugen SP, Berkmen MB, Ross W, Gaal T, Ward C, Gourse RL. 2006. rRNA promoter regulation by nonoptimal binding of $\sigma$ region 1.2: An additional recognition element for RNA polymerase. Cell 125: 1069-1082.

Helmann JD, deHaseth PL. 1999. Protein-nucleic acid interactions during open complex formation investigated by systematic alteration of the protein and DNA binding partners. Biochemistry 38: 5959-5967.

Hook-Barnard IG, Hinton DM. 2007. Transcription initiation by mix and match elements: Flexibility for polymerase binding to bacterial promoters. Gene Regul Syst Bio 1: 275-293.

Hook-Barnard IG, Hinton DM. 2009. The promoter spacer influences transcription initiation via $\sigma^{70}$ region 1.1 of Escherichia coli RNA polymerase. Proc Natl Acad Sci 106: 737-742. 
Hook-Barnard I, Johnson XB, Hinton DM. 2006. Escherichia coli RNA polymerase recognition of a $\sigma^{70}$-dependent promoter requiring a -35 DNA element and an extended -10 TGn motif. J Bacteriol 188: 8352-8359.

Juang YL, Helmann JD. 1994. A promoter melting region in the primary $\sigma$ factor of Bacillus subtilis. Identification of functionally important aromatic amino acids. I Mol Biol 235: 1470-1488.

Koo BM, Rhodius VA, Campbell EA, Gross CA. 2009a. Dissection of recognition determinants of Escherichia coli $\sigma^{32}$ suggests a composite -10 region with an 'extended $-10^{\prime}$ motif and a core -10 element. Mol Microbiol 72: 815-829.

Koo BM, Rhodius VA, Campbell EA, Gross CA. 2009b. Mutational analysis of Escherichia coli $\sigma^{28}$ and its target promoters reveals recognition of a composite -10 region, comprised of an 'extended -10 motif' and a core -10 element. Mol Microbiol 72: 830-843.

Kourennaia OV, deHaseth PL. 2007. Substitution of a highly conserved histidine in the Escherichia coli heat shock transcription factor, $\sigma^{32}$, affects promoter utilization in vitro and leads to overexpression of the biofilm-associated flu protein in vivo. J Bacteriol 189: 8430-8436.

Kumar A, Malloch RA, Fujita N, Smillie DA, Ishihama A, Hayward RS. 1993. The -35-recognition region of Escherichia coli $\sigma^{70}$ is inessential for initiation of transcription at an 'extended -10' promoter. J Mol Biol 232: 406-418.

Kusukawa N, Yura T. 1988. Heat shock protein GroE of Escherichia coli: Key protective roles against thermal stress. Genes \& Dev 2: 874-882.

Kuznedelov K, Minakhin L, Niedziela-Majka A, Dove SL, Rogulja D, Nickels BE, Hochschild A, Heyduk T, Severinov K. 2002. A role for interaction of the RNA polymerase flap domain with the $\sigma$ subunit in promoter recognition. Science 295: 855-857.

Lee SJ, Gralla JD. 2003. Open complex formation in vitro by $\sigma^{38}$ (rpoS) RNA polymerase: Roles for region 2 amino acids. J Mol Biol 329: 941-948.

Li XY, McClure WR. 1998. Characterization of the closed complex intermediate formed during transcription initiation by Escherichia coli RNA polymerase. I Biol Chem 273: 23549-23557.

Lim HM, Lee HJ, Roy S, Adhya S. 2001. A 'master' in base unpairing during isomerization of a promoter upon RNA polymerase binding. Proc Natl Acad Sci 98: 14849-14852.

Lisser S, Margalit H. 1993. Compilation of E. coli mRNA promoter sequences. Nucleic Acids Res 21: 1507-1516.

Lonetto M, Gribskov M, Gross CA. 1992. The $\sigma^{70}$ family: Sequence conservation and evolutionary relationships. I Bacteriol 174: 3843-3849.

McGrath PT, Lee H, Zhang L, Iniesta AA, Hottes AK, Tan MH, Hillson NI, Hu P, Shapiro L, McAdams HH. 2007. Highthroughput identification of transcription start sites, conserved promoter motifs and predicted regulons. Nat Biotechnol 25: 584-592.

Mecsas J, Cowing DW, Gross CA. 1991. Development of RNA polymerase-promoter contacts during open complex formation. J Mol Biol 220: 585-597.

Minakhin L, Severinov K. 2003. On the role of the Escherichia coli RNA polymerase $\sigma^{70}$ region 4.2 and $\alpha$-subunit C-terminal domains in promoter complex formation on the extended -10 galP1 promoter. J Biol Chem 278: 29710-29718.

Mitchell JE, Zheng D, Busby SJ, Minchin SD. 2003. Identification and analysis of 'extended -10 ' promoters in Escherichia coli. Nucleic Acids Res 31: 4689-4695.

Murakami KS, Darst SA. 2003. Bacterial RNA polymerases: The wholo story. Curr Opin Struct Biol 13: 31-39.
Murakami KS, Masuda S, Campbell EA, Muzzin O, Darst SA. 2002. Structural basis of transcription initiation: An RNA polymerase holoenzyme-DNA complex. Science 296: 12851290.

Nakahigashi K, Yanagi H, Yura T. 1995. Isolation and sequence analysis of rpoH genes encoding $\sigma^{32}$ homologs from gram negative bacteria: Conserved mRNA and protein segments for heat shock regulation. Nucleic Acids Res 23: 4383-4390.

Nonaka G, Blankschien M, Herman C, Gross CA, Rhodius VA. 2006. Regulon and promoter analysis of the E. coli heatshock factor, $\sigma^{32}$, reveals a multifaceted cellular response to heat stress. Genes \& Dev 20: 1776-1789.

Paget MS, Helmann JD. 2003. The $\sigma^{70}$ family of $\sigma$ factors. Genome Biol 4: 203. doi: 10.1186/gb-2003-4-1-203.

Ponnambalam S, Webster C, Bingham A, Busby S. 1986. Transcription initiation at the Escherichia coli galactose operon promoters in the absence of the normal -35 region sequences. J Biol Chem 261: 16043-16048.

Rhodius VA, Suh WC, Nonaka G, West J, Gross CA. 2006. Conserved and variable functions of the $\sigma^{\mathrm{E}}$ stress response in related genomes. PLoS Biol 4: e2. doi: 10.1371/journal. pbio.0040002.

Ross W, Gosink KK, Salomon J, Igarashi K, Zou C, Ishihama A, Severinov K, Gourse RL. 1993. A third recognition element in bacterial promoters: DNA binding by the $\alpha$ subunit of RNA polymerase. Science 262: 1407-1413.

Schneider TD, Stormo GD, Gold L, Ehrenfeucht A. 1986. Information content of binding sites on nucleotide sequences. J Mol Biol 188: 415-431.

Schroeder LA, Gries TJ, Saecker RM, Record MT Jr, Harris ME, deHaseth PL. 2009. Evidence for a tyrosine-adenine stacking interaction and for a short-lived open intermediate subsequent to initial binding of Escherichia coli RNA polymerase to promoter DNA. J Mol Biol 385: 339-349.

Serizawa M, Yamamoto H, Yamaguchi H, Fujita Y, Kobayashi K, Ogasawara N, Sekiguchi J. 2004. Systematic analysis of SigDregulated genes in Bacillus subtilis by DNA microarray and Northern blotting analyses. Gene 329: 125-136.

Shen L, Feng X, Yuan Y, Luo X, Hatch TP, Hughes KT, Liu JS, Zhang YX. 2006. Selective promoter recognition by chlamydial $\sigma^{28}$ holoenzyme. J Bacteriol 188: 7364-7377.

Shultzaberger RK, Chen Z, Lewis KA, Schneider TD. 2007. Anatomy of Escherichia coli $\sigma^{70}$ promoters. Nucleic Acids Res 35: 771-788.

Siegele DA, Hu JC, Walter WA, Gross CA. 1989. Altered promoter recognition by mutant forms of the $\sigma^{70}$ subunit of Escherichia coli RNA polymerase. J Mol Biol 206: 591-603.

Sorenson MK, Darst SA. 2006. Disulfide cross-linking indicates that FlgM-bound and free $\sigma^{28}$ adopt similar conformations. Proc Natl Acad Sci 103: 16722-16727.

Sorenson MK, Ray SS, Darst SA. 2004. Crystal structure of the flagellar $\sigma /$ anti- $\sigma$ complex $\sigma^{28} /$ FlgM reveals an intact $\sigma$ factor in an inactive conformation. Mol Cell 14: 127-138.

Tatti KM, Jones CH, Moran CP Jr. 1991. Genetic evidence for interaction of $\sigma^{\mathrm{E}}$ with the spoIIID promoter in Bacillus subtilis. J Bacteriol 173: 7828-7833.

Thouvenot B, Charpentier B, Branlant C. 2004. The strong efficiency of the Escherichia coli gapA P1 promoter depends on a complex combination of functional determinants. Biochem J 383: 371-382.

Tomsic M, Tsujikawa L, Panaghie G, Wang Y, Azok J, deHaseth PL. 2001. Different roles for basic and aromatic amino acids in conserved region 2 of Escherichia coli $\sigma^{70}$ in the nucleation and maintenance of the single-stranded DNA bubble in open RNA polymerase-promoter complexes. I Biol Chem 276: 31891-31896. 
Koo et al.

Tsujikawa L, Tsodikov OV, deHaseth PL. 2002. Interaction of RNA polymerase with forked DNA: Evidence for two kinetically significant intermediates on the pathway to the final complex. Proc Natl Acad Sci 99: 3493-3498.

Ueki T, Inouye S. 2001. SigB, SigC, and SigE from Myxococcus xanthus homologous to $\sigma^{32}$ are not required for heat shock response but for multicellular differentiation. I Mol Microbiol Biotechnol 3: 287-293.

van Schaik W, van der Voort M, Molenaar D, Moezelaar R, de Vos WM, Abee T. 2007. Identification of the $\sigma^{\mathrm{B}}$ regulon of Bacillus cereus and conservation of $\sigma^{\mathrm{B}}$-regulated genes in low-GC-content gram-positive bacteria. I Bacteriol 189: 4384-4390.

Wade JT, Roa DC, Grainger DC, Hurd D, Busby SJ, Struhl K, Nudler E. 2006. Extensive functional overlap between $\sigma$ factors in Escherichia coli. Nat Struct Mol Biol 13: 806-814.

Waldburger C, Gardella T, Wong R, Susskind MM. 1990. Changes in conserved region 2 of Escherichia coli $\sigma^{70}$ affecting promoter recognition. J Mol Biol 215: 267-276.

Young BA, Gruber TM, Gross CA. 2004. Minimal machinery of RNA polymerase holoenzyme sufficient for promoter melting. Science 303: 1382-1384.

Yu HH, Tan M. 2003. $\sigma^{28}$ RNA polymerase regulates hetB, a late developmental gene in Chlamydia. Mol Microbiol 50: 577584.

Yura T, Nakahigashi K. 1999. Regulation of the heat-shock response. Curr Opin Microbiol 2: 153-158.

Zhao K, Liu M, Burgess RR. 2005. The global transcriptional response of Escherichia coli to induced $\sigma^{32}$ protein involves $\sigma^{32}$ regulon activation followed by inactivation and degradation of $\sigma^{32}$ in vivo. J Biol Chem 280: $17758-17768$.

Zhao K, Liu M, Burgess RR. 2007. Adaptation in bacterial flagellar and motility systems: From regulon members to 'foraging'-like behavior in E. coli. Nucleic Acids Res 35: 4441-4452. 


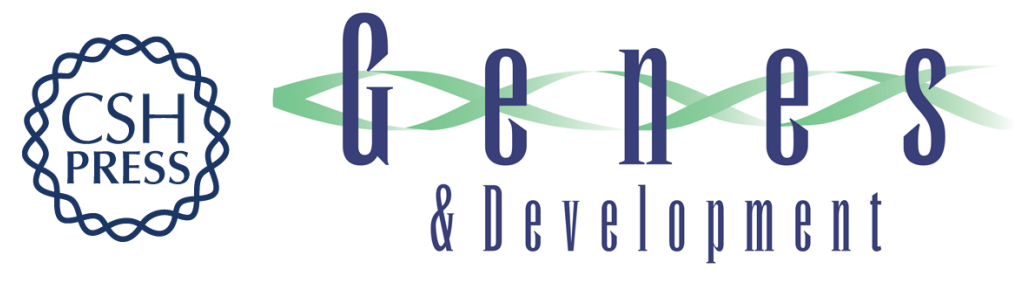

\section{Reduced capacity of alternative $\sigma s$ to melt promoters ensures stringent promoter recognition}

Byoung-Mo Koo, Virgil A. Rhodius, Gen Nonaka, et al.

Genes Dev. 2009, 23:

Access the most recent version at doi:10.1101/gad.1843709

\section{Supplemental http://genesdev.cshlp.org/content/suppl/2009/10/15/23.20.2426.DC1 \\ Material}

Related Content Promoter recognition by bacterial alternative Ã factors: the price of high selectivity?

Andrey Feklistov and Seth A. Darst

Genes Dev. October , 2009 23: 2371-2375

References This article cites 70 articles, 32 of which can be accessed free at: http://genesdev.cshlp.org/content/23/20/2426.full.html\#ref-list-1

Articles cited in:

http://genesdev.cshlp.org/content/23/20/2426.full.html\#related-urls

\section{License}

Email Alerting

Service

Receive free email alerts when new articles cite this article - sign up in the box at the top right corner of the article or click here.

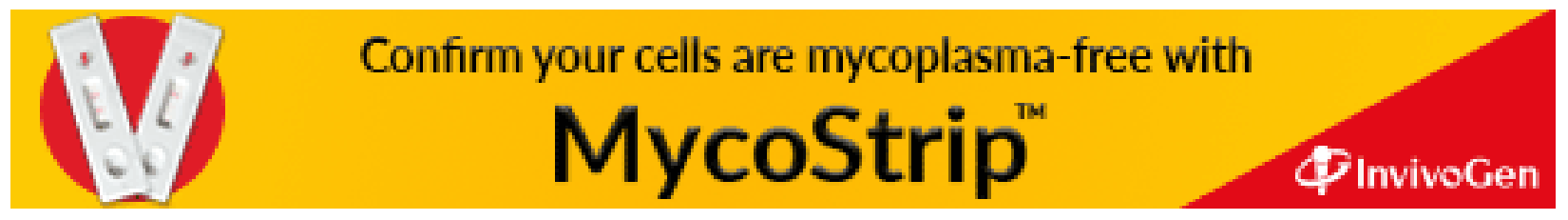

